\title{
A Diagnostic Dilemma of Cryptogenic Organising Pneumonia
}

\author{
Gurung $\mathrm{K}^{1}$ \\ 'Maidstone and Tunbridge Wells NHS Trust, Maidstone General Hospital, Maidstone, Kent, ME16 9QQ, UK.
}

\section{ABSTRACT}

Cryptogenic Organising Pneumonia is a rare lung condition, which has incidence of 6-9 cases per 1000,000 people with onset at age group between 50-60. The pathogenesis of this condition remains unknown. It mimics like pneumonia but has a good outcome with steroid treatment. Early recognition is very important and treatment with steroid therapy can save lives. This case highlights the unusual cause of shortness of breath due to COP and co existing incidental severe AS where we faced a diagnostic dilemma till lung biopsy was performed.

Keywords: Cryptogenic organising pneumonia; Pneumonia.

\section{INTRODUCTION}

Cryptogenic Organising Pneumonia (COP) is a rare lung condition, first described by $\mathrm{Dr}$ Gary Epler in $1985 .^{1}$ The incidence is $6-9$ cases per 1000,000 people with onset at age group between $50-60 .^{2}$ It is an idiopathic form of organising pneumonia previously known as idiopathic bronchiolitis obliterans organising pneumonia or BOOP. ${ }^{3}$ COP is a clinicopathological condition characterized by restrictive ventillatory defect and intra alveolar aggregation of proliferative granulation tissue on histological examination. ${ }^{4}$ The pathogenesis of this condition remains unknown. ${ }^{5}$ It mimics like pneumonia but has a good outcome with steroid treatment. ${ }^{5}$ Early recognition is very important and treatment with steroid therapy can save lives. This case highlights the unusual cause of shortness of breath due to COP and co existing incidental severe AS where we faced a diagnostic dilemma till lung biopsy was performed.

\section{CASE REPORT}

A retired quarry worker and smoker previously fit and well, presented to us in accident and emergency department with rapidly worsening shortness of breath of 4 days. He was treated by his GP for LRTI with oral amoxicillin and prednisolone prior to admission with no benefit. He gave history of gradual onset of shortness of breath of two months duration but deteriorated in the last 4 days with increasing shortness of breath, scanty whitish productive cough and occasional haemoptysis. He did not have fever, chills or rigor. There was no associated chest pain, palpitation or syncope. He denied any history of significant weight loss. Past medical illness includes paroxysmal atrial fibrillation and Bell's palsy. He was on bisoprolol $2.5 \mathrm{mg}$ once daily for AF. He is a smoker, 20/day for more than 20 years and drinks 2-3 units per week.

On examination he was breathless and tachypneic. The respiratory rate was 32 , normotensive $(130 / 5 \mathrm{mmHg})$, in atrial fibrillation with rate of 115 , apyrexial and desaturating on room air with $\mathrm{SpO}_{2} 81 \%$. $\mathrm{SpO}_{2}$ improved to $99 \%$ with high flow oxygen (rebreathing mask). Chest examination revealed diffuse bilateral crackles. There was no clubbing. Systemic examination

Correspondence: Dr. Kiran Gurung, Maidstone and Tunbridge Wells NHS Trust, Maidstone General Hospital, Maidstone, Kent, ME 16 $9 Q Q$, UK. Email: kiranprati2012@gmail.com, Phone: 07533474671. 
of CVS, abdomen and CNS were unremarkable. Chest $\mathrm{x}$ ray suggested right mid zone consolidation (Figure 1). ABG on room air showed normal pH (7.35), low $\mathrm{PaO}_{2}$ (8.1 kPa), normal $\mathrm{PaCO}_{2}(4.5 \mathrm{kPa})$, normal $\mathrm{HCO}_{3}^{-}(27$ $\mathrm{mEq} / \mathrm{L})$ and normal lactate $(1.2 \mathrm{mmol} / \mathrm{L})$. Blood results on admission showed normal haemoglobin $(13.2 \mathrm{gm} /$ $\mathrm{dL})$, leucocytosis $\left(32 \times 10^{9} / \mathrm{L}\right)$, normal platelet count, neutrophilia ( $24 \times 10^{9} /$ L), high CRP 124 , normal LFT and normal renal function test. Despite on iv antibiotic patient did not improve and chest $x$ ray was repeated on third day which showed extensive patchy shadowing in both lung fields (Figure 2). Blood and sputum culture were negative all time. Atypical pneumonia screen was negative. Avian and respiratory serology (Influenza A, B, Adenovirus, Mycoplasma, Psittacosis, Coxiella Burnetti) were insignificant. HIV and Pul TB tests were negative. PFT was impaired with FEV 1.10 (31\%), FVC 2.07 (46\%), FEV / FVC - 53\%, PEF - 83 (35\%). Subsequently HRCT scan was performed which showed ground glass appearances in both upper lobes sparing peripheries. We thought it was due to pulmonary edema. There was no evidence of volume loss. HRCT scan was repeated as there was no improvement after a month despite optimizing heart failure treatment. The previously noted ground glass opacification through the upper lobes was replaced by rapidly progressing prominent central consolidation involving lower and lingular lobes, sparing peripheries with thickening of interlobular septae (Figure $3,4)$. Bronchospcopy and bronchoalveolar lavage was not much help at this point. The autoimmune antibodies ANA, ENA, ANCA were negative. Lung biopsy was vital at this point which confirmed the diagnosis of cryptogenic organising pneumonia (Figure 5). He was started on oral Prednisolone and improved dramatically. Repeat PFT improved with steroids treatment - FEV 1.7 (48\%), FVC 2.55 (57\%), FEV / FVC 67\%, and PEF 225 (45\%). Bilateral patchy infiltrates on chest $x$ ray and HRCT cleared with treatment (Figure 6, 7). Subsequently patient underwent successful TAVI (Transcatheter aortic valve replacement) for incidental co existent critical aortic stenosis.

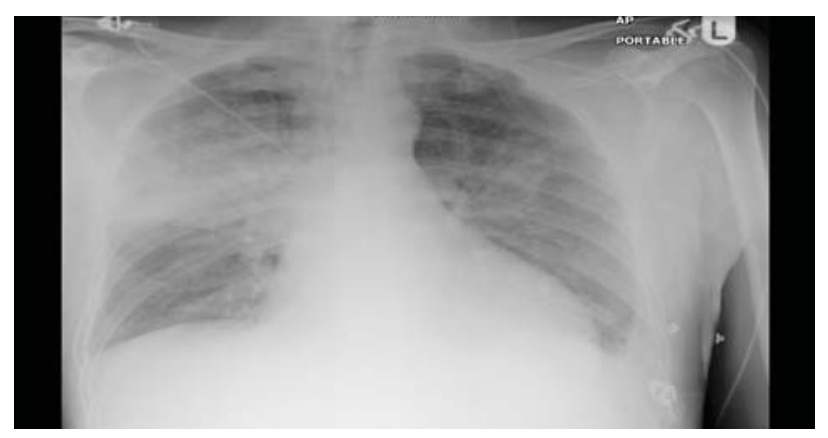

Figure 1. Arrival chest $\mathrm{x}$ ray showing right mid zone consolidation.

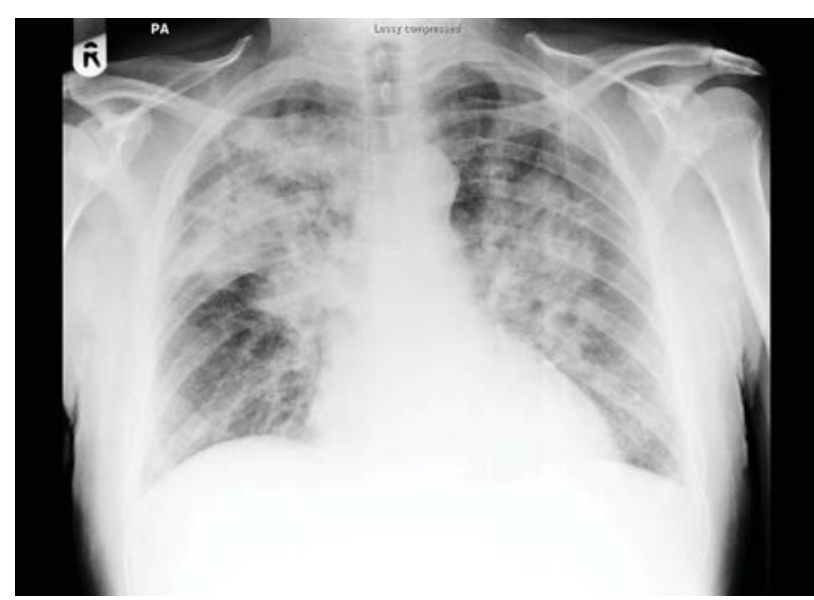

Figure 2. Repeat chest $x$ ray on third day showing extensive alveolar infiltrate in both lungs.

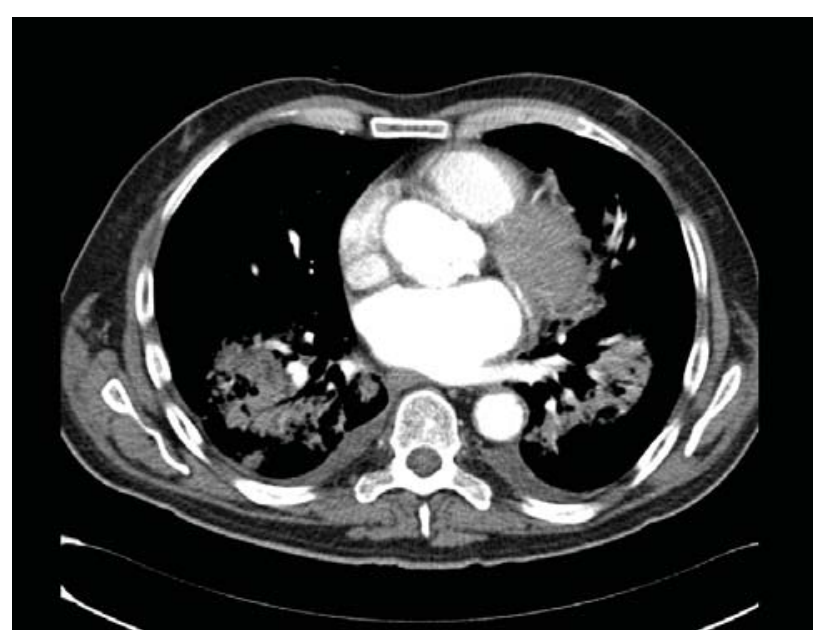

Figure 3. HRCT scan film showing prominent central consolidation.

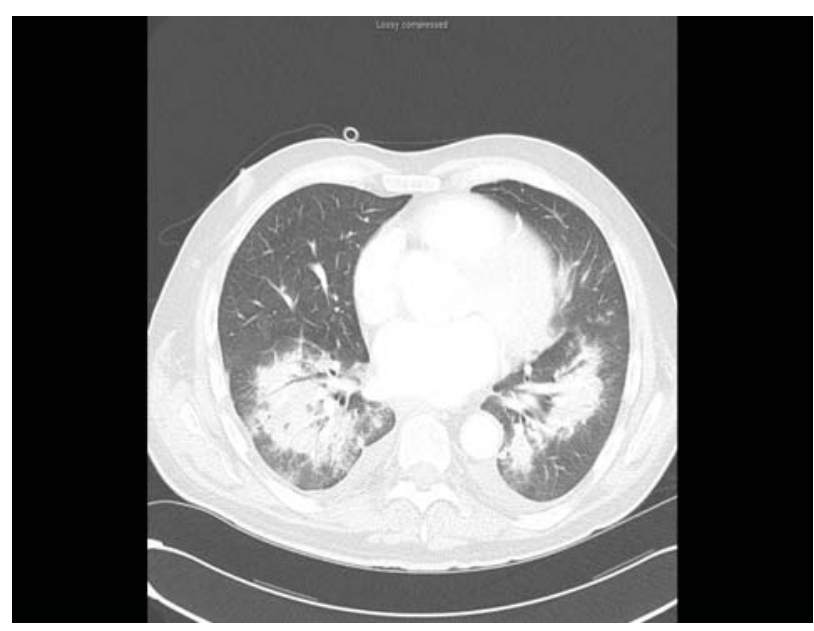

Figure 4. HRCT scan film showing prominent central consolidation. 


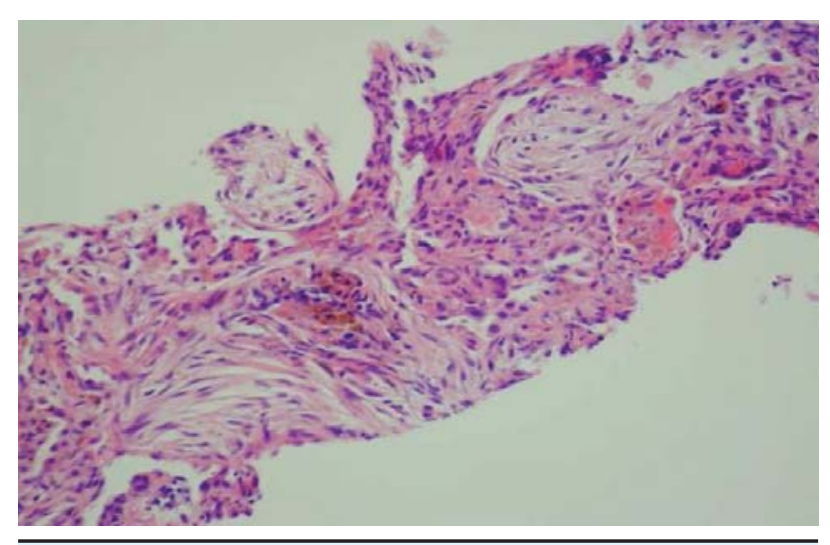

Figure 5. H\&E x 200 showing buds of intra-alveolar granulation tissue typical of an organising pneumonia.

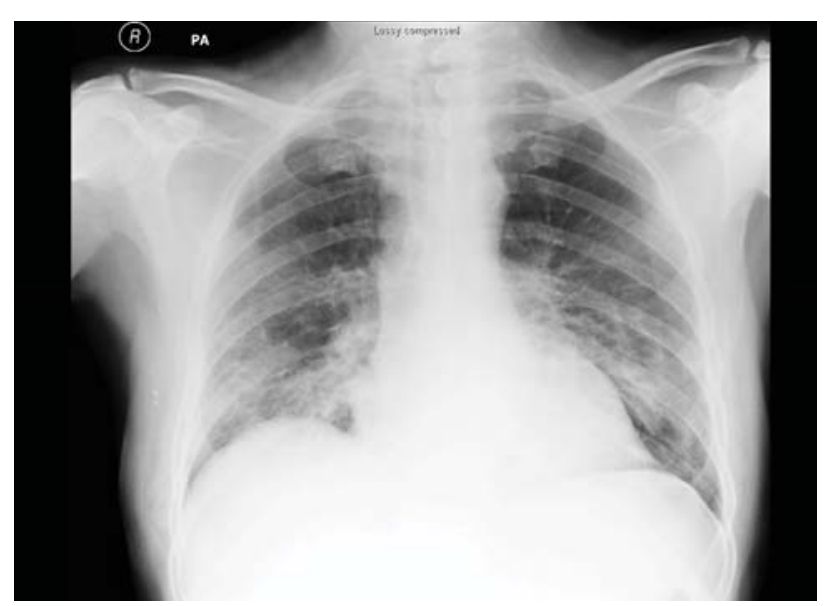

Figure 6. Repeat chest $x$ ray film after steroid treatment showing complete resolution of bilateral alveolar infiltrates.

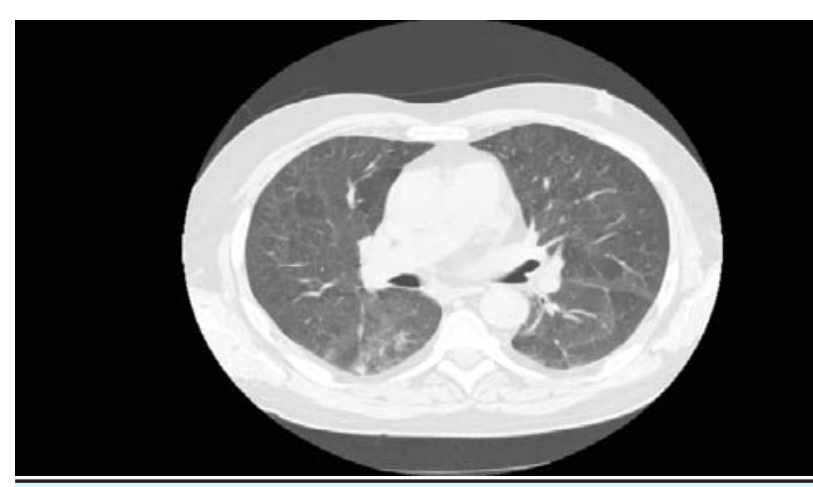

Figure 7. HRCT showing marked improvement in bilateral alveolar infiltrates after steroid treatment.

\section{DISCUSSION}

COP (Cryptogenic organizing pneumonia) is an idiopathic form of organising pneumonia. It is a rare inflammatory lung condition which equally affects men and women of age group between $50-60 .^{2}$ The inflammatory process involves both peripheral bronchioles and alveoli. It is caused by various etiologies but in half the cases, no apparent cause is found which is idiopathic. ${ }^{2-5}$ The other causes can be infections, collagen vascular diseases - SLE, rheumatoid arthritis, drugs, chemotherapy, radiotherapy, post bone marrow and organ transplant. ${ }^{2-4}$ No definite etiology was found in our patient.

The inflammatory markers were high thus it could have been post infectious but cultures and special stains did not show any organisms. Raised CRP, leucocytosis and neutrophilia are seen in organising pneumonia. They can mimic flu like symptoms with cough, fever, malaise, fatigue and dyspnea. ${ }^{2-4} 75 \%$ of patients will have symptoms of less than 2 months. Only few people will have symptoms less than six month before diagnosis. ${ }^{2-4}$ This patient had gradual onset shortness of breath on exertion for two months before becoming acutely more breathless at rest for four days before arriving in accident and emergency department.

The only physical findings can be crackles on auscultation and hypoxaemia on pulse oximetry and ABG which was found in this patient. Diagnosis is based on chest $x$ ray, chest HRCT scan, PFT and lung biopsy. ${ }^{3,4}$ The Chest $x$ ray can be distinctive to extensive pneumonia, with bilateral consolidation or simple unilateral consolidation. ${ }^{6}$ our patient had right upper zone consolidation at admission but shadow progressed to become widespread involving both lungs in three days. The consolidation patch migrates from one zone to other as disease progresses which was found in our case. ${ }^{6}$ Small nodular opacities may be seen in chest $x$ ray in $50 \%$ and large nodule in $15 \%$ patients. ${ }^{3,4,6} \mathrm{~A}$ ground glass appearance with consolidation is seen in most patients. Bronchoscopy and brochoalveolar lavage will show upto $40 \%$ lymphocytes and subtle neutrophils and eosinphils. ${ }^{2-4}$

Biopsy showing typical organizing pneumonia without any other feature suggesting other conditions confirms the diagnosis. It responds with steroid treatment. Prednisolone is initiated at $1.0-1.5 \mathrm{mg} / \mathrm{kg} / \mathrm{day}$ to a maximum of $100 \mathrm{mg}$ a day given as a single dose in the morning. It is recommended this dose is maintained for 4-8 weeks. If the patient is improved or stable, the prednisolone dose is gradually tapered to $0.5-1 \mathrm{mg} / \mathrm{kg}$ for another 4-6 weeks. It is tapered to zero after 3-6 months if patient remains stable or improved. ${ }^{2,5}$ 
Gurung. A Diagnostic Dilemma of Cryptogenic Organising Pneumonia.

\section{REFERENCES}

1. Epler GR, Colby TV, Mcloud TC, Carrington CB, Gaensler EA. Bronchiolitis obliterans organising pneumonia. N Engl J Med. 1985;312:152-8.

2. Talmadge EK. Cryptogenic organising pneumonia. [Online] USA: Uptodate; 2011. Available from: htttp://www. uptodate.com [Accessed 25th Feb 2012].

3. Cordier JF. Cryptogenic organizing pneumonia. Eur Respir J. 2006;28(2):422-46.
4. Epler GR. Bronchiolitis obliterans organising pneumonia. Arch Intern Med. 2001;161(2):158-64.

5. Epler GR. Bronchiolitis obliterans organising pneumonia, 25 years: a variety of causes but what are the treatment options? Expert Rev Respir Med. 2011;5(3)353-61.

6. Oymak FS, Demirbas HM, Mavili E, Akgun H,Gulmez I,Demir I,Ozesmi M. Bronchiolitis obliterans organizing pneumonia: Clinical and roentgenological features in 26 Cases. Respiration. 2005;72(3):254-62. 